QUARTERLY OF APPLIED MATHEMATICS

VOLUME LXIV, NUMBER 3

SEPTEMBER 2006, PAGES 529-546

$\mathrm{S}$ 0033-569X(06)01022-4

Article electronically published on June 15, 2006

\title{
SOME REMARKS ON HOMOGENIZATION AND EXACT BOUNDARY CONTROLLABILITY FOR THE ONE-DIMENSIONAL WAVE EQUATION
}

\author{
BY \\ PABLO PEDREGAL (Departamento de Matemáticas, ETSI Industriales, Universidad de Castilla-La \\ Mancha, 13071 Ciudad Real, Spain) \\ AND \\ FRANCISCO PERIAGO (Departamento de Matemática Aplicada y Estadistica, ETSI Industriales, \\ Universidad Politécnica de Cartagena, 30203 Cartagena, Spain)
}

\begin{abstract}
This paper contains three results concerning the homogenization and exact controllability for the one-dimensional wave equation. First, we give sufficient conditions on the initial data to ensure the convergence of the conormal derivatives associated with the wave equation with a rapidly oscillating coefficient and zero Dirichlet boundary conditions. Secondly, we apply this result to prove the existence of a class of initial data whose associated boundary controls are uniformly bounded and obtain some information (in particular, its limit behavior) on this class of data. Finally, we prove that all initial data in $L^{2} \times H^{-1}$ may be uniformly controlled but at the price of adding an internal feedback control in our system. The main advantage of this last procedure is that we have explicit formulae for both states and controls.
\end{abstract}

1. Introduction. As the title indicates, this work contains a few remarks on the homogenization and uniform exact boundary controllability for the one-dimensional wave equation.

Let us briefly describe these remarks in this introduction. Consider first the homogeneous system

$$
\left(A^{\varepsilon}\right) \begin{cases}\phi_{t t}^{\varepsilon}-\left(a^{\varepsilon}(x) \phi_{x}^{\varepsilon}\right)_{x}=0, & 0<x<1, t>0 \\ \phi^{\varepsilon}(0, t)=\phi^{\varepsilon}(1, t)=0, & t>0 \\ \phi^{\varepsilon}(x, 0)=\phi_{0}^{\varepsilon}(x), \quad \phi_{t}^{\varepsilon}(x, 0)=\phi_{1}^{\varepsilon}(x), & 0<x<1,\end{cases}
$$

Received November 30, 2005.

2000 Mathematics Subject Classification. Primary 35B27, 35L05, 93B05.

The first author was supported by project MTM2004-07114 from Ministerio de Educación y Ciencia (Spain) and PAI05-029 from JCCM (Castilla-La Mancha, Spain).

The second author was supported by projects MTM2004-07114 from Ministerio de Educación y Ciencia (Spain) and 00675/PI/04 from Fundación Séneca (Murcia, Spain).

E-mail address: pablo.pedregal@uclm.es

E-mail address: f.periago@upct.es

(C)2006 Brown University 
where for $\varepsilon$ positive, $a^{\varepsilon}(x)=a(x / \varepsilon), a$ being a 1-periodic function which satisfies

$$
0<a_{m} \leq a(x) \leq a_{M}<\infty \quad \text { a.e. } x \in \mathbb{R},
$$

and the initial data $\left(\phi_{0}^{\varepsilon}, \phi_{1}^{\varepsilon}\right)$ are taken in the usual energy space $H_{0}^{1} \times L^{2}$.

As is well known, the main goal of homogenization theory is to try to understand the limit behavior, as the parameter $\varepsilon$ goes to zero, of some quantities (the solution $\phi^{\varepsilon}(x, t)$, the energy of such a solution, etc.) associated with this system. Among these quantities, in controllability theory it is of particular interest to analyze the behavior of the conormal derivatives of a solution at one of the extremes which are given by

$$
f^{\varepsilon}(t)=\left(a^{\varepsilon} \phi_{x}^{\varepsilon}\right)(1, t) .
$$

This quantity also has a physical meaning when the system $\left(A^{\varepsilon}\right)$ models the vibrations of a string, namely, (1.1) represents the string tension at the extreme $x=1$ and at time $t$.

Under the natural hypothesis of weak convergence of the initial data, the first basic homogenization result concerning the system $\left(A^{\varepsilon}\right)$ ensures the weak* convergence in $L^{\infty}\left(0, T ; H_{0}^{1}\right)$ of the solution $\phi^{\varepsilon}(x, t)$ to a function $\phi(x, t)$ which solves the simpler constant-coefficient system

$$
(A) \begin{cases}\phi_{t t}-a^{0} \phi_{x x}=0, & 0<x<1, t>0, \\ \phi(0, t)=\phi(1, t)=0, & t>0, \\ \phi(x, 0)=\phi_{0}(x), \quad \phi_{t}(x, 0)=\phi_{1}(x), & 0<x<1,\end{cases}
$$

where $a^{0}=\left(\int_{0}^{1} \frac{1}{a(s)} d s\right)^{-1}$ is the homogenized coefficient and $\left(\phi_{0}, \phi_{1}\right)$ are the weak limits in $H_{0}^{1} \times L^{2}$ of $\left(\phi_{0}^{\varepsilon}, \phi_{1}^{\varepsilon}\right)$.

However, this hypothesis of weak convergence of the initial data is not enough to ensure the convergence of some other important quantities associated with the system $\left(A^{\varepsilon}\right)$, for instance its energy, to the corresponding quantity of the limit system $(A)$ (see [8. Chapter 12]). More regularity and suitable convergence properties on the initial data are needed.

Something similar happens with the conormal derivatives (1.1). It is known (see [4]) that for $T>0$ there exist uniformly bounded sequences of initial data $\left(\phi_{0}^{\varepsilon}, \phi_{1}^{\varepsilon}\right) \in$ $H_{0}^{1}(0,1) \times L^{2}(0,1)$ for which (1.1) is not uniformly bounded in $L^{2}(0, T)$. So, as in the case of the energy, it is quite natural to look for sufficient conditions on the initial data $\left(\phi_{0}^{\varepsilon}, \phi_{1}^{\varepsilon}\right)$ in order to ensure the convergence of (1.1) to the corresponding derivatives of the homogenized system $(A)$. This will be our first task in this work. Precisely, in Section 2 we analyze in detail this question and give sufficient conditions on the initial data $\left(\phi_{0}^{\varepsilon}, \phi_{1}^{\varepsilon}\right)$ to ensure the convergence

$$
\left(a^{\varepsilon} \phi_{x}^{\varepsilon}\right)(1, \cdot) \rightarrow a^{0} \phi_{x}(1, \cdot)
$$

both weak and strong in $L^{2}(0, T)$. This is our Theorem 2.1.

In the second part of this paper, we focus on the control system

$$
\left(C^{\varepsilon}\right) \begin{cases}u_{t t}^{\varepsilon}-\left(a^{\varepsilon} u_{x}^{\varepsilon}\right)_{x}=0, & 0<x<1, t>0, \\ u^{\varepsilon}(0, t)=0, \quad u^{\varepsilon}(1, t)=f^{\varepsilon}(t), & t>0, \\ u^{\varepsilon}(x, 0)=u_{0}^{\varepsilon}(x), \quad u_{t}^{\varepsilon}(x, 0)=u_{1}^{\varepsilon}(x) & 0<x<1,\end{cases}
$$


where the objective now is to find a time $T>0$, independent of $\varepsilon$, and a family of boundary controls $f^{\varepsilon}(t)$ such that for each $\varepsilon>0$ and initial states $\left(u_{0}^{\varepsilon}, u_{1}^{\varepsilon}\right)$ in appropriate spaces, the solution $u^{\varepsilon}(x, t)$ satisfies the uniform exact controllability condition

$$
u^{\varepsilon}(x, T)=u_{t}^{\varepsilon}(x, T)=0 \quad \text { for all } x \in(0,1) .
$$

Under suitable regularity assumptions on the function $a$ and on the initial data, this problem has a positive answer as shown for instance in [10]. However, in [2] the existence was observed of bounded sequences of initial data $\left(u_{0}^{\varepsilon_{j}}, u_{1}^{\varepsilon_{j}}\right) \in L^{2}(0,1) \times H^{-1}(0,1)$ which cannot be controlled with a sequence of controls $f^{\varepsilon_{j}} \in L^{2}(0, T)$, of minimal $L^{2}$-norm, uniformly bounded in $\varepsilon_{j}$. From this, it is deduced that the convergence of the family of controls $f^{\varepsilon_{j}}$ does not take place even in a weak sense.

A partial cure for the pathology of lack of convergence of the boundary controls was suggested in [4, where two partial controllability results for the projection of the solutions over the subspaces generated by the eigenfunctions with wavelengths longer and shorter than $\varepsilon$ were established. In other words, what is uniformly controlled in [4] is not the whole solution $u^{\varepsilon}$ but the truncation of this solution at low and high frequencies. Then, the convergence is proved of the family of controls to the corresponding control of the associated homogenized system in the case of low frequencies, and to zero in the high frequency case. These two results were derived from some uniform observability inequalities for the adjoint system which are obtained after a careful spectral analysis of the indicated adjoint system (see [6, 7]). The extension of these results to the case of several space dimensions and low frequencies can be found in [11.

In Section 3, we revisit this problem with the aim of giving positive convergence results for the states and controls of system $\left(C^{\varepsilon}\right)$ to the corresponding states and controls of its associated homogenized system.

First, as an immediate consequence of the positive result of convergence of the conormal derivatives, we deduce the existence of a class of initial data for which the associated boundary controls are uniformly bounded; for this class of data (which from now on we will call the class of nonresonant initial data) we have nice convergence properties of the states and controls of the system $\left(C^{\varepsilon}\right)$ to the corresponding states and controls of the associated homogenized system (Theorem 3.1). We are also able to obtain some information on this class of initial data, in particular, its limit behavior as $\varepsilon \rightarrow 0$.

Secondly, and once we know it is not possible to control uniformly all the initial data in $L^{2} \times H^{-1}$ from the extreme $x=1$, we analyze what seems to be quite natural to overcome this difficulty, that is, to add more control elements in our system. In this way, we will see that adding an appropriate feedback control acting on the whole of the string we have nice properties of convergence of states and controls for all initial data in $L^{2} \times H^{-1}$. This is our Theorem 3.3. The main advantage of this procedure is that it provides us with explicit formulae for the states and controls, which is of major importance from a practical point of view. This result also has some similarity with what happens with the numerical schemes for the computation of the exact control of the linear constant-coefficient wave equation where it is also necessary to introduce an additional term, which also acts as an internal control (see [17]), in order to filter out the high frequency spurious oscillations introduced by the numerical scheme. 
Finally, we conclude the paper with a short section on comments and related open problems.

2. Convergence of the conormal derivatives. Let us consider the system $\left(A^{\varepsilon}\right)$ with initial data $\left(\phi_{0}^{\varepsilon}, \phi_{1}^{\varepsilon}\right)$ satisfying the following conditions:

$\begin{cases}(i) & \phi_{0}^{\varepsilon} \in H^{2}(0,1) \cap H_{0}^{1}(0,1) \text { is uniformly bounded in } H^{2}(0,1) ; \\ \text { (ii) } & \left(a^{\varepsilon}\left(\phi_{0}^{\varepsilon}\right)^{\prime}\right)^{\prime} \rightarrow\left(a^{0} \phi_{0}^{\prime}\right)^{\prime} \quad \text { strongly in } H^{-1}(0,1) \text { for some } \phi_{0} \in H_{0}^{1}(0,1) ; \\ (\text { iii }) & \phi_{1}^{\varepsilon} \in H_{0}^{1}(0,1) \text { is uniformly bounded in } H_{0}^{1}(0,1) .\end{cases}$

As we will see later on in this section, these three hypotheses are enough to prove the convergence of the conormal derivatives (1.1) to the corresponding derivatives of its associated homogenized system, but before proving it a few comments on the above conditions are in order:

- Conditions (ii) and (iii) were introduced by Brahim-Otsmane, Francfort and Murat in their paper [3] on the corrector results for the wave equation, and they are sufficient conditions for the convergence of the energy of the system $\left(A^{\varepsilon}\right)$ to the energy of $(A)$. As we will see in the proof below, in our result on the convergence of the conormal derivatives we also need condition $(i)$ to obtain some uniform bounds on the term $\phi_{t t}^{\varepsilon}$ which leads to a uniform estimate on (1.1) in $L^{2}(0, T)$.

- In order for assumptions $(i)$ and (ii) to be compatible, we must necessarily have $\phi_{0}=0$. To see this, we first note that by the Rellich-Kondrachov theorem there exists a subsequence, still denoted by $\varepsilon$, such that

$$
\phi_{0}^{\varepsilon} \rightarrow \overline{\phi_{0}} \quad \text { strongly in } H^{1}(0,1) .
$$

On the other hand, assumption (ii) implies (see [8, pp. 236-238] for the details) that

$$
\phi_{0}^{\varepsilon} \rightarrow \phi_{0} \quad \text { weakly in } H^{1}(0,1) .
$$

Therefore, $\phi_{0}=\overline{\phi_{0}}$. Moreover, condition (ii) also implies the convergence of the energy

$$
\int_{0}^{1} a^{\varepsilon}(x)\left(\phi_{0}^{\varepsilon}\right)^{\prime}(x)\left(\phi_{0}^{\varepsilon}\right)^{\prime}(x) d x \rightarrow \int_{0}^{1} a^{0} \phi_{0}^{\prime}(x) \phi_{0}^{\prime}(x) d x,
$$

but by the strong convergence in (2.1),

$$
\int_{0}^{1} a^{\varepsilon}(x)\left(\phi_{0}^{\varepsilon}\right)^{\prime}(x)\left(\phi_{0}^{\varepsilon}\right)^{\prime}(x) d x \rightarrow \int_{0}^{1} \bar{a} \phi_{0}^{\prime}(x) \phi_{0}^{\prime}(x) d x,
$$

where $\bar{a}=\int_{0}^{1} a(s) d s$. So, the only possibility for (2.2) and (2.3) to be equal is $\phi_{0}^{\prime}=0$. As $\phi_{0} \in H_{0}^{1}(0,1)$, we conclude that $\phi_{0}=0$.

- A very simple example of sequences satisfying assumptions $(i)$ and (ii) occurs when $\left\{\phi_{0}^{\varepsilon}\right\}_{0<\varepsilon<1}$ has the form

$$
\phi_{0}^{\varepsilon}(x)=\varepsilon^{\alpha} \varphi(x), \quad \text { for } \alpha>0 \text { and } \varphi \in H^{2}(0,1) \cap H_{0}^{1}(0,1) .
$$


Theorem 2.1. Fix $T>0$. Let $a \in B V(0,1)$ and denote also by $a$ the 1-periodic extension of this function to all of $\mathbb{R}$. Assume that

$$
0<a_{m} \leq a(x) \leq a_{M}<\infty \text { a.e. } x \in \mathbb{R}
$$

and set $a^{\varepsilon}(x)=a(x / \varepsilon)$, with $0<\varepsilon<1$. Consider the homogeneous system $\left(A^{\varepsilon}\right)$ with initial data satisfying (i), (ii) and (iii) above. Then, by passing to a subsequence still denoted by $\varepsilon$, we have

$$
\left(a^{\varepsilon} \phi_{x}^{\varepsilon}\right)(1, \cdot) \rightarrow a^{0} \phi_{x}(1, \cdot) \quad \text { weakly in } L^{2}(0, T),
$$

$\phi$ being the solution of the homogenized problem

$$
\left(A^{0}\right) \begin{cases}\phi_{t t}-a^{0} \phi_{x x}=0, & 0<x<1, t>0, \\ \phi(0, t)=\phi(1, t)=0, & t>0, \\ \phi(x, 0)=0, \quad \phi_{t}(x, 0)=\phi_{1}(x), & 0<x<1,\end{cases}
$$

where $\phi_{1}$ is the strong limit in $L^{2}(0,1)$ of (a subsequence of) $\phi_{1}^{\varepsilon}$.

Moreover, if the initial data satisfy the following additional regularity conditions

$$
\left\{\begin{array}{l}
\text { (iv) } \phi_{0}^{\varepsilon} \in H^{3}(0,1) \text { is uniformly bounded in } H^{3}(0,1), \\
\text { (v) }\left(a^{\varepsilon}\left(\phi_{0}^{\varepsilon}\right)^{\prime}\right)^{\prime} \in H_{0}^{1}(0,1), \text { and } \\
\text { (vi) } \phi_{1}^{\varepsilon} \in H^{2}(0,1) \text { is uniformly bounded in } H^{2}(0,1)
\end{array}\right.
$$

then the convergence in (2.4) is strong in $L^{2}(0, T)$, that is,

$$
\left(a^{\varepsilon} \phi_{x}^{\varepsilon}\right)(1, \cdot) \rightarrow a^{0} \phi_{x}(1, \cdot) \quad \text { strongly in } L^{2}(0, T)
$$

Proof. By our hypotheses $(i)$ and $(i i i)$ on the initial data, we can conclude (see 9 , Th. 5 (ii), p. 389]) that $\phi_{t t}^{\varepsilon}$ belongs to $L^{2}(0,1)$ for each fixed time $t$, and

$$
\left\|\phi_{t t}^{\varepsilon}\right\|_{L^{\infty}\left(0, T ; L^{2}(0,1)\right)} \leq C\left(\left\|\phi_{0}^{\varepsilon}\right\|_{H^{2}}+\left\|\phi_{1}^{\varepsilon}\right\|_{H_{0}^{1}}\right) \leq C\left(c_{1}+c_{2}\right),
$$

with the constants $C, c_{1}$ and $c_{2}$ independent of $\varepsilon$. In addition, thanks to hypothesis (ii), we can apply Theorem 12.10 of $[8]$ to obtain the convergence

$$
\phi_{t}^{\varepsilon} \rightarrow \phi_{t} \quad \text { in } C\left([0, T] ; L^{2}(0,1)\right) .
$$

At this point we notice that the above convergence (2.7) holds if the initial data $\phi_{1}^{\varepsilon}$ satisfy

$$
\phi_{1}^{\varepsilon} \rightarrow \phi_{1} \quad \text { strongly in } L^{2}(0,1),
$$

but, by the Rellich-Kondrachov theorem and by passing to a subsequence, this is a direct consequence of our hypothesis (iii) .

It is elementary to compute explicitly that

$$
\left(a^{\varepsilon} \phi_{x}^{\varepsilon}\right)(1, t)=a^{0} \int_{0}^{1} \frac{1}{a(s / \varepsilon)} F^{\varepsilon}(s, t) d s-F^{\varepsilon}(1, t),
$$

where

$$
F^{\varepsilon}(x, t)=-\int_{0}^{x} \phi_{t t}^{\varepsilon}(s, t) d s \in H^{1}(0,1)
$$

for each fixed time $t$. To deduce this, simply notice that we are looking at $-\phi_{t t}^{\varepsilon}$ as a given right-hand side in the wave equation. 
Notice first that, because of (2.6) and the representation in (2.8), the sequence $\left\{\phi_{x}^{\varepsilon}(1, t)\right\}$ is uniformly bounded in $L^{2}(0, T)$. Then, for any $0<a<b<T$, we have

$$
\int_{a}^{b} F^{\varepsilon}(1, t) d t=-\int_{0}^{1} \int_{a}^{b} \phi_{t t}^{\varepsilon}(s, t) d t d s=\int_{0}^{1}\left[\phi_{t}^{\varepsilon}(s, a)-\phi_{t}^{\varepsilon}(s, b)\right] d s,
$$

and by the strong convergence in (2.7),

$$
\int_{a}^{b} F^{\varepsilon}(1, t) d t \rightarrow \int_{0}^{1}\left[\phi_{t}(s, a)-\phi_{t}(s, b)\right] d s .
$$

This means that the convergence occurs towards the field $F$, which is defined for the homogenized equation just as $F^{\varepsilon}$ has been defined for the $\varepsilon$-th equation. For the other term in (2.8), a similar computation yields

$$
\int_{a}^{b} \int_{0}^{1} \frac{1}{a(s / \varepsilon)} F^{\varepsilon}(s, t) d s d t=\int_{0}^{1} \int_{0}^{s} \frac{1}{a(s / \varepsilon)}\left[\phi_{t}^{\varepsilon}(r, a)-\phi_{t}^{\varepsilon}(r, b)\right] d r d s .
$$

Once again, due to the strong convergence in (2.7) and to the weak convergence $1 / a^{\varepsilon} \rightarrow$ $1 / a^{0}$, we can conclude the convergence of the previous integrals to the same integrals replacing $F^{\varepsilon}$ by $F$ as indicated before.

Finally, from our additional hypotheses $(i v)-(v i)$, it is deduced (see [9. Th. 6, p. 391]) that $\phi_{t t t}^{\varepsilon} \in L^{\infty}\left(0, T ; L^{2}(0,1)\right)$ and

$$
\left\|\phi_{t t t}^{\varepsilon}\right\|_{L^{\infty}\left(0, T ; L^{2}(0,1)\right)} \leq \widetilde{C}
$$

with $\widetilde{C}$ independent of $\varepsilon$. Then, by writing

$$
\frac{d}{d t}\left(a^{\varepsilon} \phi_{x}^{\varepsilon}\right)(1, t)=a^{0} \int_{0}^{1} \frac{1}{a(s / \varepsilon)} F_{t}^{\varepsilon}(s, t) d s-F_{t}^{\varepsilon}(1, t)
$$

we easily obtain that $\frac{d}{d t}\left(a^{\varepsilon} \phi_{x}^{\varepsilon}\right)(1, t)$ is uniformly bounded in $L^{2}(0, T)$. Again, by the Rellich-Kondrachov theorem and by passing to a subsequence one deduces that the convergence in (2.4) is strong in $L^{2}(0, T)$.

3. A pair of uniform exact controllability results. Next, we deal with the control system $\left(C^{\varepsilon}\right)$. We will divide our analysis on the convergence properties of the family of controls $f^{\varepsilon}(t)$ into two parts. In the first one, we will prove that for a particular class of initial data the resonance phenomena on the controls (which are present when the initial data are taken in $L^{2}(0,1) \times H^{-1}(0,1)$; see [2]) disappear, and we will try to obtain some information on this class of nonresonant initial data. In the second one, we will add more control elements in our system in order to control uniformly all the initial data.

3.1. Uniformly controlling a particular class of initial data. When we face a controllability problem such as $\left(C^{\varepsilon}\right)$ it is much more convenient to look first at the simpler adjoint system $\left(A^{\varepsilon}\right)$. The reason is that uniform exact controllability for $\left(C^{\varepsilon}\right)$ is equivalent to some well-known uniform observability inequality for the solutions of $\left(A^{\varepsilon}\right)$. Moreover, the HUM method (see [13] for a complete description of this method) establishes that when 
exact controllability for the system $\left(C^{\varepsilon}\right)$ holds, the boundary control $f^{\varepsilon}(t)$ of minimal $L^{2}(0, T)$-norm has the form

$$
f^{\varepsilon}(t)=\left(a^{\varepsilon} \phi_{x}^{\varepsilon}\right)(1, t)
$$

for some appropriate initial data $\left(\phi_{0}^{\varepsilon}, \phi_{1}^{\varepsilon}\right)$ of the adjoint system $\left(A^{\varepsilon}\right)$. Therefore, the convergence properties of the family of controls (3.1) are linked to the convergence properties of the conormal derivatives of the associated adjoint system. In particular, from every sequence of initial data $\left(\phi_{0}^{\varepsilon}, \phi_{1}^{\varepsilon}\right)$ of the adjoint system $\left(A^{\varepsilon}\right)$ for which we have convergence of its conormal derivatives we obtain a sequence of initial data $\left(u_{0}^{\varepsilon}, u_{1}^{\varepsilon}\right)$ of the system $\left(C^{\varepsilon}\right)$ for which we have both exact controllability in a finite time and uniform boundedness (in particular, weak convergence) of its controls. Precisely, we have the following result.

Theorem 3.1. Let $a^{\varepsilon}$ be as in Theorem 2.1. Then, for any $T \geq 2\left(a^{0}\right)^{-1 / 2}$ there exist nontrivial, uniformly bounded sequences of initial data

$$
\left(u_{0}^{\varepsilon}, u_{1}^{\varepsilon}\right) \in L^{2}(0,1) \times H^{-1}(0,1)
$$

and of controls $f^{\varepsilon} \in L^{2}(0, T)$ such that the solution $u^{\varepsilon}(x, t)$ of the system $\left(C^{\varepsilon}\right)$ satisfies

$$
u^{\varepsilon}(x, T)=u_{t}^{\varepsilon}(x, T)=0, \quad 0<x<1 .
$$

Moreover, by passing to a subsequence still denoted by $\varepsilon$, the sequences of states $u^{\varepsilon}$ and of controls $f^{\varepsilon}$ of problem $\left(C^{\varepsilon}\right)$ satisfy

$$
\left\{\begin{array}{l}
u^{\varepsilon} \rightarrow u \quad \text { strongly in } L^{2}\left(0, T ; L^{2}(0,1)\right), \\
f_{\varepsilon} \rightarrow f \quad \text { strongly in } L^{2}(0, T)
\end{array}\right.
$$

$u$ and $f$ being, respectively, the state and control of the homogenized system

$$
\left(C^{0}\right) \begin{cases}u_{t t}-a^{0} u_{x x}=0, & 0<x<1, t>0, \\ u(0, t)=0, \quad u(1, t)=f(t), & t>0 \\ u(x, 0)=u_{0}(x), \quad u_{t}(x, 0)=u_{1}(x), & 0<x<1,\end{cases}
$$

where $\left(u_{0}, u_{1}\right)$ are the weak limits in $L^{2}(0,1) \times H^{-1}(0,1)$ of (a subsequence of) $\left(u_{0}^{\varepsilon}, u_{1}^{\varepsilon}\right)$.

Proof. The strategy to show our result comes directly from the HUM and is quite standard in controllability theory, but is included here for convenience. We proceed in 3 steps.

Step 1. Definition and uniform boundedness of the sequences $\left(u_{0}^{\varepsilon}, u_{1}^{\varepsilon}\right)$. Consider the adjoint system $\left(A^{\varepsilon}\right)$ with initial data satisfying conditions $(i)-(v i i)$ above. Then for any $\varepsilon>0$ the functions

$$
f^{\varepsilon}(t)=\left(a^{\varepsilon} \phi_{x}^{\varepsilon}\right)(1, t),
$$

$\phi^{\varepsilon}(x, t)$ being the solution of $\left(A^{\varepsilon}\right)$, provide the controls to lead the system $\left(C^{\varepsilon}\right)$ to rest at time $T$ and for the sequence of initial data $\left(u_{0}^{\varepsilon}, u_{1}^{\varepsilon}\right)$ just taken as the "final" states of the backward problem

$$
\begin{cases}y_{t t}^{\varepsilon}-\left(a^{\varepsilon} y_{x}^{\varepsilon}\right)_{x}=0, & 0<x<1,0<t<T, \\ y^{\varepsilon}(0, t)=0 ; y^{\varepsilon}(1, t)=f^{\varepsilon}(t), & 0<t<T, \\ y^{\varepsilon}(x, T)=y_{t}^{\varepsilon}(x, T)=0, & 0<x<1,\end{cases}
$$


that is,

$$
u_{0}^{\varepsilon}(x)=y^{\varepsilon}(x, 0) \quad \text { and } \quad u_{1}^{\varepsilon}(x)=y_{t}^{\varepsilon}(x, 0) .
$$

Note that since $a \in B V(0,1)$, as shown in [10, Th. 3.1], this system is well-posed in the space $L^{2}(0,1) \times H^{-1}(0,1)$, that is, there is a unique solution $y^{\varepsilon}(x, t)$ in the class

$$
C\left([0, T] \times L^{2}(0,1)\right) \cap C^{1}\left([0, T] \times H^{-1}(0,1)\right) .
$$

In fact, there is a very precise relationship between the initial data $\left(\phi_{0}^{\varepsilon}, \phi_{1}^{\varepsilon}\right)$ and $\left(u_{0}^{\varepsilon}, u_{1}^{\varepsilon}\right)$, namely,

$$
\Lambda^{\varepsilon}\left(\phi_{0}^{\varepsilon}, \phi_{1}^{\varepsilon}\right)=\left(u_{1}^{\varepsilon},-u_{0}^{\varepsilon}\right)
$$

where $\Lambda^{\varepsilon}$ is defined by

$$
\begin{aligned}
& \left\langle\Lambda^{\varepsilon}\left(\phi_{0}^{\varepsilon}, \phi_{1}^{\varepsilon}\right),\left(\theta_{0}, \theta_{1}\right)\right\rangle \\
& =\left\langle\left(u_{1}^{\varepsilon},-u_{0}^{\varepsilon}\right),\left(\theta_{0}, \theta_{1}\right)\right\rangle \\
& =\left\langle u_{1}^{\varepsilon}, \theta_{0}\right\rangle_{H^{-1} \times H_{0}^{1}}-\int_{0}^{1} u_{0}^{\varepsilon}(x) \theta_{1}(x) d x \\
& =\int_{0}^{T} a^{\varepsilon}(1) f^{\varepsilon}(t) \theta_{x}^{\varepsilon}(1, t) d t,
\end{aligned}
$$

$\theta^{\varepsilon}(x, t)$ being the solution of the system

$$
\left(B^{\varepsilon}\right) \begin{cases}\theta_{t t}^{\varepsilon}-\left(a^{\varepsilon} \theta_{x}^{\varepsilon}\right)_{x}=0, & 0<x<1, t>0 \\ \theta^{\varepsilon}(0, t)=\theta^{\varepsilon}(1, t)=0, & t>0 \\ \theta^{\varepsilon}(x, 0)=\theta_{0}(x), \quad \theta_{t}^{\varepsilon}(x, 0)=\theta_{1}(x), & 0<x<1,\end{cases}
$$

with $\left(\theta_{0}, \theta_{1}\right) \in H_{0}^{1} \times L^{2}$. Now take $\theta_{0}=0$ and $\theta_{1} \in \mathcal{D}(0,1)$. Then, by the preceding lemma, the sequences $\phi_{x}^{\varepsilon}(1, \cdot)$ and $\theta_{x}^{\varepsilon}(1, \cdot)$ are uniformly bounded in $L^{2}(0, T)$ and therefore the sequence

$$
\int_{0}^{1} u_{0}^{\varepsilon}(x) \theta_{1}(x) d x=-\int_{0}^{T} a^{\varepsilon}(1) f^{\varepsilon}(t) \theta_{x}^{\varepsilon}(1, t) d t
$$

is bounded. In fact, by the Hahn-Banach theorem, the above still holds for $\theta_{1} \in$ $L^{2}(0,1)$. It follows, by the Banach-Steinhaus theorem, that the sequence $\left\{u_{0}^{\varepsilon}\right\}$ is uniformly bounded in $L^{2}$.

In order to prove that the sequence $\left\{u_{1}^{\varepsilon}\right\}$ is uniformly bounded in $H^{-1}(0,1)$ we take $\theta_{1}=0$ and $\theta_{0} \in \mathcal{D}(0,1)$. Thus, by [9, Th. 5 (ii), p. 389] and by using the representation in (2.8) for the function $\theta_{x}^{\varepsilon}(1, t)$, we obtain that the sequence $\theta_{x}^{\varepsilon}(1, \cdot)$ is uniformly bounded in $L^{2}(0, T)$. We then proceed as above by using the identity

$$
\left\langle u_{1}^{\varepsilon}, \theta_{0}\right\rangle_{H^{-1} \times H_{0}^{1}}=\int_{0}^{T} a^{\varepsilon}(1) f^{\varepsilon}(1, t) \theta_{x}^{\varepsilon}(1, t) d t .
$$

Step 2. Identification of the limit system and convergence of the controls. From the HUM it is deduced that $a^{0} \phi_{x}(1, t)$ is the control of the constant coefficient system

$$
(C) \begin{cases}\bar{u}_{t t}-a^{0} \bar{u}_{x x}=0, & 0<x<1, t>0, \\ \bar{u}(0, t)=0, \quad \bar{u}(1, t)=a^{0} \phi_{x}(1, t), & t>0, \\ \bar{u}(x, 0)=\bar{u}_{0}(x), \quad \bar{u}_{t}(x, 0)=\bar{u}_{1}(x), & 0<x<1,\end{cases}
$$


where the initial data of this system are obtained through the HUM isomorphism $\Lambda$, namely,

$$
\Lambda\left(0, \phi_{1}\right)=\left(\bar{u}_{1},-\bar{u}_{0}\right)
$$

Moreover, we have the identity

$$
\left\langle\bar{u}_{1}, \theta_{0}\right\rangle_{H^{-1} \times H_{0}^{1}}-\int_{0}^{1} \bar{u}_{0} \theta_{1} d x=\int_{0}^{T}\left(a^{0}\right)^{2} \phi_{x}(1, t) \theta_{x}(1, t) d t,
$$

for any solution $\theta(x, t)$ of the system

$$
\left(B^{0}\right) \begin{cases}\theta_{t t}-a^{0} \theta_{x x}=0, & 0<x<1, t>0, \\ \theta(0, t)=\theta(1, t)=0, & t>0, \\ \theta(x, 0)=\theta_{0}(x), \quad \theta_{t}(x, 0)=\theta_{1}(x), & 0<x<1,\end{cases}
$$

with $\left(\theta_{0}, \theta_{1}\right) \in H_{0}^{1}(0,1) \times L^{2}(0,1)$.

On the other hand, as the sequences $\left(u_{0}^{\varepsilon}, u_{1}^{\varepsilon}\right)$ are uniformly bounded in $L^{2} \times H^{-1}$, by passing to a subsequence,

$$
\left(u_{0}^{\varepsilon}, u_{1}^{\varepsilon}\right) \rightarrow\left(u_{0}, u_{1}\right) \quad \text { weakly in } L^{2} \times H^{-1} .
$$

Now consider the controllability problem $\left(C^{0}\right)$ associated with these initial data. We want to show that problems $(C)$ and $\left(C^{0}\right)$ are equivalent, that is, that

$$
\left(u_{0}, u_{1}\right)=\left(\bar{u}_{0}, \bar{u}_{1}\right) \text { and } f(t)=a^{0} \phi_{x}(1, t) .
$$

We proceed by transposition. Let us consider a solution $\theta^{\varepsilon}(x, t)$ of problem $\left(B^{\varepsilon}\right)$ with initial data $\theta^{0}=0$ and $\theta^{1} \in \mathcal{D}(0,1)$. Multiplying $\theta^{\varepsilon}$ by the wave equation in $\left(A^{\varepsilon}\right)$ and integrating by parts we get (3.3). Taking limits in this identity, from the convergence (2.5) and (3.5), it follows that

$$
\int_{0}^{T}\left(a^{0}\right)^{2} \phi_{x}(1, t) \theta_{x}(1, t) d t=-\int_{0}^{1} u_{0}(x) \theta_{1}(x) d x
$$

where $\theta$ is a solution of $\left(B^{0}\right)$. Similarly, multiplying $\theta^{\varepsilon}$ by the wave equation in $(C)$ and integrating by parts, we arrive at the identity

$$
\int_{0}^{T} a(1) a^{0} \phi_{x}(1, t) \theta_{x}^{\varepsilon}(1, t) d t+\int_{0}^{T} \int_{0}^{1}\left(a^{\varepsilon}-a^{0}\right) \bar{u}_{x} \theta_{x}^{\varepsilon} d x d t=-\int_{0}^{1} \bar{u}_{0} \theta_{1} d x .
$$

Taking limits in this identity as $\varepsilon \rightarrow 0$ and using a classical homogenization result (see [8. Th. 12.6]), i.e.,

$$
\int_{0}^{T} \int_{0}^{1}\left(a^{\varepsilon}-a^{0}\right) \bar{u}_{x}(x, t) \theta_{x}^{\varepsilon}(x, t) d x d t \rightarrow 0
$$

we obtain

$$
\int_{0}^{T}\left(a^{0}\right)^{2} \phi_{x}(1, t) \theta_{x}(1, t) d t=-\int_{0}^{1} \bar{u}_{0}(x) \theta_{1}(x) d x .
$$

Thus, since (3.6) and (3.7) hold for any $\theta_{1} \in \mathcal{D}(0,1)$, we conclude that $u_{0}=\bar{u}_{0}$.

In order to prove that $u_{1}=\bar{u}_{1}$, let us consider system $\left(B^{\varepsilon}\right)$ with initial data $\theta_{0} \in$ $\mathcal{D}(0,1)$ and $\theta_{1}=0$. Then, taking limits as $\varepsilon \rightarrow 0$ in (3.4) and by passing to a subsequence, we get

$$
\int_{0}^{T} a(1) a^{0} \phi_{x}(1, t) h(t) d t=\left\langle u_{1}, \theta_{0}\right\rangle_{H^{-1} \times H_{0}^{1}}
$$


where $h(t)$ is the weak limit in $L^{2}(0, T)$ of a subsequence of $\theta_{x}^{\varepsilon}(1, t)$. Note that we cannot ensure that $h(t)=\theta_{x}(1, t)$ since $\theta_{0}$ does not satisfy assumption $(i i)$.

Arguing as before, we also have

$$
\int_{0}^{T} a(1) a^{0} \phi_{x}(1, t) \theta_{x}^{\varepsilon}(1, t) d t+\int_{0}^{T} \int_{0}^{1}\left(a^{\varepsilon}-a^{0}\right) \bar{u}_{x} \theta_{x}^{\varepsilon} d x d t=\left\langle\bar{u}_{1}, \theta_{0}\right\rangle_{H^{-1} \times H_{0}^{1}}
$$

and, taking limits in this expression,

$$
\int_{0}^{T} a(1) a^{0} \phi_{x}(1, t) h(t) d t=\left\langle\bar{u}_{1}, \theta_{0}\right\rangle_{H^{-1} \times H_{0}^{1}} .
$$

From (3.8) and (3.9), we see that $u_{1}=\bar{u}_{1}$.

Finally, due to the uniqueness of the HUM controls of the systems $\left(C^{0}\right)$ and $(C)$ we easily obtain

$$
f(t)=a^{0} \phi_{x}(1, t)
$$

The convergence of the controls $f^{\varepsilon}(t)$ to $f(t)$ has already been proved in Theorem 2.1.

Step 3. Convergence of the states. We proceed again by transposition. For a given $g \in L^{2}\left(0, T ; L^{2}(0,1)\right)$ we consider the system

$$
\begin{cases}\theta_{t t}^{\varepsilon}-\left(a^{\varepsilon} \theta_{x}^{\varepsilon}\right)_{x}=g, & 0<x<1, t>0, \\ \theta^{\varepsilon}(0, t)=\theta^{\varepsilon}(1, t)=0, & t>0, \\ \theta^{\varepsilon}(x, 0)=0, \quad \theta_{t}^{\varepsilon}(x, 0)=0, & 0<x<1 .\end{cases}
$$

Writing

$$
F^{\varepsilon}(x, t)=\int_{0}^{x}\left[-\phi_{t t}^{\varepsilon}(s, t)+g(s, t)\right] d s
$$

and following along the lines of the proof of Theorem 2.1, we arrive at the convergence

$$
\left(a \theta_{x}^{\varepsilon}\right)(1, t) \rightarrow a^{0} \theta_{x}(1, t) \quad \text { strongly in } L^{2}(0, T),
$$

where $\theta(x, t)$ is the solution of the associated homogenized system

$$
\begin{cases}\theta_{t t}-\left(a^{0} \theta_{x}\right)_{x}=g, & 0<x<1, t>0, \\ \theta(0, t)=\theta(1, t)=0, & t>0, \\ \theta(x, 0)=0, \quad \theta_{t}(x, 0)=0, & 0<x<1 .\end{cases}
$$

Multiplying the wave equation in $\left(C^{\varepsilon}\right)$ by $\theta^{\varepsilon}$ and integrating by parts, we easily obtain the identity

$$
\int_{0}^{T} \int_{0}^{1} u^{\varepsilon} g d x d t=-\int_{0}^{T} a(1) f^{\varepsilon}(t) \theta_{x}^{\varepsilon}(1, t) d t .
$$

Due to the strong convergence in $L^{2}(0, T)$ of $f^{\varepsilon} \rightarrow f$ and $\left(a \theta_{x}^{\varepsilon}\right)(1, \cdot) \rightarrow a^{0} \theta_{x}(1, \cdot)$, by passing to the limit as $\varepsilon \rightarrow 0$ in (3.11), we get

$$
\int_{0}^{T} \int_{0}^{1} \bar{u} g d x d t=-\int_{0}^{T} a^{0} f(t) \theta_{x}(1, t) d t
$$

for some $\bar{u} \in L^{2}\left(0, T ; L^{2}(0,1)\right)$. Indeed, $\bar{u}=u$ is the solution of the associated homogenized system since both $u^{\varepsilon}$ and $u$ are characterized by relations (3.11) and (3.12) for all $g \in L^{2}\left(0, T ; L^{2}(0, T)\right)$. This proves the weak convergence in $L^{2}\left(0, T ; L^{2}(0,1)\right)$ of $u^{\varepsilon}$ to $u$. 
In order to show that this convergence is in fact strong, it suffices to prove that for any sequence $g^{\varepsilon} \in L^{2}\left(0, T ; L^{2}(0,1)\right)$ with

$$
g^{\varepsilon} \rightarrow g \quad \text { weakly in } L^{2}\left(0, T ; L^{2}(0,1)\right)
$$

we have

$$
\int_{0}^{T} \int_{0}^{1} u^{\varepsilon} g^{\varepsilon} d x d t \rightarrow \int_{0}^{T} \int_{0}^{1} u g d x d t
$$

Reasoning as before, it is not difficult to obtain the identity

$$
\int_{0}^{T} \int_{0}^{1} u^{\varepsilon} g^{\varepsilon} d x d t=-\int_{0}^{T} a(1) f^{\varepsilon}(t) \theta_{x}^{\varepsilon}(1, t) d t
$$

where now $\theta^{\varepsilon}$ is the solution of system (3.10) with $g$ replaced by $g^{\varepsilon}$. Since the convergence $g^{\varepsilon} \rightarrow g$ is only weak, we cannot apply Theorem 12.10 of [8] (which needs a strong convergence) to show, as in Theorem 2.1, that $\theta_{x}^{\varepsilon}(1, t) \rightarrow \theta_{x}(1, t)$ weakly in $L^{2}(0, T), \theta$ being the solution of the associated homogenized system. However, the weak convergence (3.13) implies that $g^{\varepsilon}$ is bounded in $L^{2}\left(0, T ; L^{2}(0,1)\right)$ and hence, as in the proof of Theorem 2.1 by passing to a subsequence still denoted by $\varepsilon$, one concludes that

$$
\theta_{x}^{\varepsilon}(1, \cdot) \rightarrow h(\cdot) \quad \text { weakly in } L^{2}(0, T)
$$

for some $h \in L^{2}(0, T)$. Passing to the limit as $\varepsilon \rightarrow 0$ in (3.14),

$$
\int_{0}^{T} \int_{0}^{1} u^{\varepsilon} g^{\varepsilon} d x d t \rightarrow-\int_{0}^{T} a(1) f(t) h(t) d t .
$$

On the other hand, multiplying $\theta^{\varepsilon}$, the solution of system (3.10) with $g$ replaced by $g^{\varepsilon}$, by the wave equation of the homogenized system $\left(C^{0}\right)$ and integrating by parts we get

$$
\int_{0}^{T} \int_{0}^{1} u g^{\varepsilon} d x d t+\int_{0}^{T} \int_{0}^{1}\left(a^{\varepsilon}-a^{0}\right) u_{x} \theta_{x}^{\varepsilon} d x d t=-\int_{0}^{T} a(1) f(t) \theta_{x}^{\varepsilon}(1, t) d t .
$$

Again by a classical homogenization result (see [8, Th. 12.6]), the second term in the left-hand side of this expression converges to zero as $\varepsilon \rightarrow 0$. Hence, due to the weak convergences $g^{\varepsilon} \rightarrow g$ and $\theta_{x}^{\varepsilon}(1, \cdot) \rightarrow h(\cdot)$,

$$
\int_{0}^{T} \int_{0}^{1} u g d x d t=-\int_{0}^{T} a(1) f(t) h(t) d t
$$

which completes the proof.

Once we have proved the existence of a class of initial data for the system $\left(C^{\varepsilon}\right)$ for which the corresponding boundary controls are uniformly bounded, the natural question we must address is the characterization of this class, that is,

$(Q)\left\{\begin{array}{l}\text { given the initial data }\left(u_{0}^{\varepsilon}, u_{1}^{\varepsilon}\right) \in L^{2}(0,1) \times H^{-1}(0,1) \text {, how do we } \\ \text { know if the sequence of controls } f^{\varepsilon}(t) \text { is uniformly bounded? }\end{array}\right.$

Unfortunately, we will not give a complete answer to this question, but we will obtain some partial information on it.

To this end, we first analyze the following procedure: (1) Take $\left(\phi_{0}^{\varepsilon}, \phi_{1}^{\varepsilon}\right) \in H_{0}^{1}(0,1) \times$ $L^{2}(0,1)$ satisfying conditions $(i)-(i i)-(i i i)$ above and converging to some element $\left(0, \phi_{1}\right)$. Then, we know that $f^{\varepsilon}(t)=\left(a^{\varepsilon} \phi_{x}^{\varepsilon}\right)(1, t)$ converges to $f(t)=a^{0} \phi_{x}(1, t)$, where $\phi(x, t)$ is the solution of the homogenized system $\left(A^{0}\right)$. Moreover, $f^{\varepsilon}(t)$ and $f(t)$ are, 
respectively, the exact controls of the systems $\left(C^{\varepsilon}\right)$ and $\left(C^{0}\right)$ for some initial data $\left(u_{0}^{\varepsilon}, u_{1}^{\varepsilon}\right)$ and $\left(u_{0}, u_{1}\right)$ that, up to now, are unknown. (2) With the same initial data $\left(\phi_{0}^{\varepsilon}, \phi_{1}^{\varepsilon}\right)$ consider the constant-coefficient system

$$
\left(A_{0}^{\varepsilon}\right) \begin{cases}\psi_{t t}^{\varepsilon}-a^{0} \psi_{x x}^{\varepsilon}=0, & 0<x<1, t>0, \\ \psi^{\varepsilon}(0, t)=\psi^{\varepsilon}(1, t)=0, & t>0, \\ \psi^{\varepsilon}(x, 0)=\phi_{0}^{\varepsilon}(x), \quad \psi_{t}^{\varepsilon}(x, 0)=\phi_{1}^{\varepsilon}(x), & 0<x<1 .\end{cases}
$$

The same argument as in the case of the system $\left(A^{\varepsilon}\right)$ shows that $a^{0} \psi_{x}^{\varepsilon}(1, t)$ converges to $a^{0} \psi_{x}(1, t)$, where $\psi(x, t)$ solves the limit system

$$
\left(A_{0}\right) \begin{cases}\psi_{t t}-a^{0} \psi_{x x}=0, & 0<x<1, t>0, \\ \psi(0, t)=\psi(1, t)=0, & t>0, \\ \psi(x, 0)=0, \quad \psi_{t}(x, 0)=\phi_{1}(x), & 0<x<1 .\end{cases}
$$

Note that the systems $\left(A^{0}\right)$ and $\left(A_{0}\right)$ are equal and therefore $\phi_{x}(1, t)=\psi_{x}(1, t)$. As before, $a^{0} \psi_{x}^{\varepsilon}(1, t)$ and $a^{0} \psi_{x}(1, t)$ are the exact controls of the systems

$$
\left(C_{0}^{\varepsilon}\right) \begin{cases}v_{t t}^{\varepsilon}-a^{0} v_{x x}^{\varepsilon}=0, & 0<x<1, t>0, \\ v^{\varepsilon}(0, t)=0, \quad v^{\varepsilon}(1, t)=a^{0} \psi_{x}^{\varepsilon}(1, t), & t>0, \\ v^{\varepsilon}(x, 0)=v_{0}^{\varepsilon}(x), \quad v_{t}^{\varepsilon}(x, 0)=v_{1}^{\varepsilon}(x), & 0<x<1,\end{cases}
$$

and

$$
\left(C_{0}\right) \begin{cases}v_{t t}-a^{0} v_{x x}=0, & 0<x<1, t>0, \\ v(0, t)=0, \quad v(1, t)=a^{0} \psi_{x}(1, t), & t>0, \\ v(x, 0)=v_{0}(x), \quad v_{t}(x, 0)=v_{1}(x), & 0<x<1,\end{cases}
$$

for some appropriate couple of initial data $\left(v_{0}^{\varepsilon}, v_{1}^{\varepsilon}\right),\left(v_{0}, v_{1}\right)$, respectively. Since $\left(A^{0}\right)=$ $\left(A_{0}\right)$, we conclude that $\left(C^{0}\right)=\left(C_{0}\right)$, due to the uniqueness of the HUM control. In particular,

$$
\left(u_{0}, u_{1}\right)=\left(v_{0}, v_{1}\right) .
$$

The main advantage of considering systems $\left(A_{0}^{\varepsilon}\right)-\left(A_{0}\right)$ and $\left(C_{0}^{\varepsilon}\right)-\left(C_{0}\right)$ is that since they are constant-coefficient systems, the controls $a^{0} \psi_{x}^{\varepsilon}(1, t)$ and $a^{0} \psi_{x}(1, t)$ may be calculated explicitly via d'Alembert's formula (see next subsection). Once we have these controls, the initial data $\left(v_{0}^{\varepsilon}, v_{1}^{\varepsilon}\right)$ and $\left(v_{0}, v_{1}\right)$ may also be calculated. All we have to do is to solve, for $T=2\left(a^{0}\right)^{-1 / 2}$, the backward wave systems

$$
\begin{cases}v_{t t}^{\varepsilon}-a^{0} v_{x x}^{\varepsilon}=0, & 0<x<1, t>0, \\ v^{\varepsilon}(0, t)=0, \quad v^{\varepsilon}(1, t)=a^{0} \psi_{x}^{\varepsilon}(1, t), & t>0 \\ v^{\varepsilon}(x, T)=v_{t}^{\varepsilon}(x, T)=0, & 0<x<1\end{cases}
$$

and

$$
\begin{cases}v_{t t}-a^{0} v_{x x}=0, & 0<x<1, t>0, \\ v(0, t)=0, \quad v(1, t)=a^{0} \psi_{x}(1, t), & t>0, \\ v(x, T)=v_{t}(x, T)=0, & 0<x<1,\end{cases}
$$

and so

$$
\left(v_{0}^{\varepsilon}, v_{1}^{\varepsilon}\right)=\left(v^{\varepsilon}(x, 0), v_{t}^{\varepsilon}(x, 0)\right) \quad \text { and } \quad\left(v_{0}, v_{1}\right)=\left(v(x, 0), v_{t}(x, 0)\right) \text {. }
$$


This procedure has two important consequences:

1. We are able to determine the limit initial data $\left(u_{0}, u_{1}\right)$ corresponding to a nonresonant sequence of the initial data $\left(u_{0}^{\varepsilon}, u_{1}^{\varepsilon}\right)$ of the initial control system $\left(C^{\varepsilon}\right)$.

2. Although we do not know explicitly the sequence $\left(u_{0}^{\varepsilon}, u_{1}^{\varepsilon}\right)$, at least we know that if it is nonresonant, then $\left(u_{0}^{\varepsilon}, u_{1}^{\varepsilon}\right)$ must be not far from a sequence of the form $\left(v_{0}^{\varepsilon}, v_{1}^{\varepsilon}\right)$, which can be calculated explicitly. This means that

$$
\left(u_{0}^{\varepsilon}-v_{0}^{\varepsilon}, u_{1}^{\varepsilon}-v_{1}^{\varepsilon}\right) \rightarrow 0 \quad \text { weakly in } L^{2} \times H^{-1}
$$

because both sequences $\left(u_{0}^{\varepsilon}, u_{1}^{\varepsilon}\right)$ and $\left(v_{0}^{\varepsilon}, v_{1}^{\varepsilon}\right)$ have the same limit.

In the preceding argument our starting point was the initial data of the adjoint system $\left(A^{\varepsilon}\right)$, but in practice we should begin with initial data in the control system $\left(C^{\varepsilon}\right)$. From this new starting point, the above procedure reads as follows:

(a) Take some initial data $\left(v_{0}^{\varepsilon}, v_{1}^{\varepsilon}\right) \in L^{2} \times H^{-1}$ converging to some $\left(u_{0}, u_{1}\right)$ and solve in time $T=2\left(a^{0}\right)^{-1 / 2}$ the control problem

$$
\begin{cases}v_{t t}^{\varepsilon}-a^{0} v_{x x}^{\varepsilon}=0, & 0<x<1, t>0, \\ v^{\varepsilon}(0, t)=0, \quad v^{\varepsilon}(1, t)=g^{\varepsilon}(t), & t>0, \\ v^{\varepsilon}(x, 0)=v_{0}^{\varepsilon}(x), \quad v_{t}^{\varepsilon}(x, 0)=v_{1}^{\varepsilon}(x), & 0<x<1 .\end{cases}
$$

As mentioned before, by using the d'Alembert formula we can easily solve this problem and therefore obtain an explicit formula for the control $g^{\varepsilon}(t)$ in terms of $\left(v_{0}^{\varepsilon}, v_{1}^{\varepsilon}\right)$.

(b) With this value of $g^{\varepsilon}(t)$, solve the following inverse problem: find $\left(\phi_{0}^{\varepsilon}, \phi_{1}^{\varepsilon}\right) \in$ $H_{0}^{1} \times L^{2}$ such that the solution $\phi^{\varepsilon}(x, t)$ of the system

$$
\begin{cases}\phi_{t t}^{\varepsilon}-a^{0} \phi_{x x}^{\varepsilon}=0, & 0<x<1, t>0, \\ \phi^{\varepsilon}(0, t)=\phi^{\varepsilon}(1, t)=0, & t>0, \\ \phi^{\varepsilon}(x, 0)=\phi_{0}^{\varepsilon}(x), \quad \phi_{t}^{\varepsilon}(x, 0)=\phi_{1}^{\varepsilon}(x), & 0<x<1,\end{cases}
$$

satisfies

$$
a^{0} \phi_{x}^{\varepsilon}(1, t)=g^{\varepsilon}(t) \text { for all } 0 \leq t \leq T .
$$

This can also be done, for instance, by Fourier analysis.

(c) Finally, if $\left(\phi_{0}^{\varepsilon}, \phi_{1}^{\varepsilon}\right)$ satisfies conditions $(i)-(i i)-($ iii $)$ above, we then may put these as initial conditions for the adjoint system $\left(A^{\varepsilon}\right)$. It then produces some other initial data $\left(u_{0}^{\varepsilon}, u_{1}^{\varepsilon}\right)$ for which the corresponding control is uniformly bounded. We cannot explicitly determine the sequence $\left(u_{0}^{\varepsilon}, u_{1}^{\varepsilon}\right)$, but we know that

$$
\left(u_{0}^{\varepsilon}-v_{0}^{\varepsilon}, u_{1}^{\varepsilon}-v_{1}^{\varepsilon}\right) \rightarrow 0 \quad \text { weakly in } L^{2} \times H^{-1}
$$

and that

$$
\left(u_{0}^{\varepsilon}, u_{1}^{\varepsilon}\right) \rightarrow\left(u_{0}, u_{1}\right) \quad \text { weakly in } L^{2} \times H^{-1} .
$$

From the above, it seems to be quite possible that the data $\left(v_{0}^{\varepsilon}, v_{1}^{\varepsilon}\right)$ may be nonresonant in the sense that the associated boundary controls are uniformly bounded, but, at present, we do not know if this is true or not. 
3.2. Uniformly controlling all the initial data in $L^{2} \times H^{-1}$. Next, we will analyze the possibility of introducing an additional control element in our system in order to uniformly control all the initial data in $L^{2} \times H^{-1}$. As we will see later on, this control comes in the form of an internal control, which may be calculated explicitly. But, before doing this, we first collect some well-known results on the exact controllability for the wave equation with constant coefficients that we will need in the following. Consider the system

$$
\begin{cases}u_{t t}-c^{2} u_{x x}=0, & 0<x<b, t>0, \\ u(0, t)=0, \quad u(b, t)=f(t), & t>0, \\ u(x, 0)=u_{0}(x), \quad u_{t}(x, 0)=u_{1}(x), & 0<x<b,\end{cases}
$$

with $c>0$ constant and initial data $\left(u_{0}, u_{1}\right) \in L^{2}(0,1) \times H^{-1}(0,1)$. It is known (see for instance [15] or [12, Th. 5.1]) that for $T=2 b / c$ there exists a unique boundary control $f$ of minimal $L^{2}(0, T)$-norm which drives the solution $u$ of system (3.16) to rest at time $T$, that is,

$$
u(x, T)=u_{t}(x, T)=0, \quad 0<x<b .
$$

In particular, this control coincides with the one obtained by HUM. Indeed, there is an explicit formula for the control $f$ which can be deduced from the d'Alembert formula, namely,

$$
f(t)= \begin{cases}\frac{1}{2} u_{0}(b-c t)+\frac{1}{2 c} \int_{b-c t}^{b} u_{1}(s) d s, & 0<t<b / c, \\ -\frac{1}{2} u_{0}(c t-b)+\frac{1}{2 c} \int_{c t-b}^{b} u_{1}(s) d s, & b / c<t<2 b / c .\end{cases}
$$

The following technical remark is also in order.

REMARK 3.2. Let $\varphi \in W^{1, \infty}(0,1)$ be such that $\varphi^{-1} \in W^{1, \infty}(0, \varphi(1))$. It is well known (see [14, Lemma 3.2, p. 66]) that given $\phi \in H_{0}^{1}(0, \varphi(1))$, the element $\phi \circ \varphi \in H_{0}^{1}(0,1)$. Let $u \in H^{-1}(0,1)$ and assume that $\varphi^{\prime} u \in H^{-1}(0,1)$. By $u \circ \varphi^{-1}$ we understand the element of $H^{-1}(0, \varphi(1))$ defined by

$$
\left\langle u \circ \varphi^{-1}, \phi\right\rangle_{H^{-1}(0, \varphi(1)) \times H_{0}^{1}(0, \varphi(1))}=\left\langle\varphi^{\prime} u, \phi \circ \varphi\right\rangle_{H^{-1}(0,1) \times H_{0}^{1}(0,1)} .
$$

Finally, we recall that if $u \in H^{-1}(0,1)$, then, in the distributional sense, $u=f^{\prime}$ for a certain function $f \in L^{2}(0,1)$ which is, up to a constant, unique. So, by $\int_{a}^{b} u(s) d s$ we mean $f(b)-f(a)$.

Another interesting remark we would like to point out here is that the controllability properties of the wave equations

$$
u_{t t}^{\varepsilon}-\left(a^{\varepsilon}(x) u_{x}^{\varepsilon}\right)_{x}=0
$$

and

$$
\rho^{\varepsilon}(x) u_{t t}^{\varepsilon}-u_{x x}^{\varepsilon}=0
$$

are equivalent. This is due to the fact that a classical change of variables which we will use in the sequel transforms equation (3.18) into (3.19) and vice versa. So, for simplicity, from now on we will consider equation (3.19). We now may state and prove our last controllability result. 
Theorem 3.3. Let $\rho \in W^{1, \infty}(\mathbb{R})$ be a 1-periodic function satisfying

$$
0<\rho_{m} \leq \rho(x) \leq \rho_{M}<\infty, \quad \text { a.e. } x \in \mathbb{R},
$$

and put $\rho^{*}=\int_{0}^{1} \sqrt{\rho(s)} d s$. Then, for any $T>2 \rho^{*}, 0<\varepsilon<\varepsilon_{0}$, with $\varepsilon_{0}$ small enough, and

$$
\left(u_{0}, u_{1}\right) \in L^{2}(0,1) \times H^{-1}(0,1),
$$

there exists a control $f^{\varepsilon} \in L^{2}(0, T)$ such that the solution of the problem

$$
\begin{cases}2 \rho^{\varepsilon} u_{t t}^{\varepsilon}-u_{x x}=\rho^{\varepsilon}\left(\frac{1}{\rho^{\varepsilon}} u_{x}^{\varepsilon}\right)_{x}, & 0<x<1, t>0, \\ u^{\varepsilon}(0, t)=0, \quad u^{\varepsilon}(1, t)=f^{\varepsilon}(t), & t>0, \\ u^{\varepsilon}(x, 0)=u_{0}(x), \quad u_{t}^{\varepsilon}(x, 0)=u_{1}(x), & 0<x<1,\end{cases}
$$

satisfies the uniform exact controllability condition (1.2). Moreover,

$$
\begin{cases}u^{\varepsilon} \rightarrow u & \text { strongly in } L^{\infty}\left(0, T ; L^{2}(0,1)\right), \\ f^{\varepsilon} \rightarrow f & \text { strongly in } L^{2}(0, T),\end{cases}
$$

where $u$ and $f$ are, respectively, the state and control of the homogeneous system

$$
\begin{cases}\left(\rho^{*}\right)^{2} u_{t t}-u_{x x}=0, & 0<x<1, t>0 \\ u(0, t)=0, \quad u(1, t)=f(t), & t>0, \\ u(x, 0)=u_{0}(x), \quad u_{t}(x, 0)=u_{1}(x), & 0<x<1\end{cases}
$$

which also satisfies the exact controllability condition

$$
u(x, T)=u_{t}(x, T)=0 \quad \text { for all } x \in(0,1) .
$$

Proof. Note that since $\rho \in W^{1, \infty}(\mathbb{R})$, equation

$$
2 \rho^{\varepsilon} u_{t t}^{\varepsilon}-u_{x x}=\rho^{\varepsilon}\left(\frac{1}{\rho^{\varepsilon}} u_{x}^{\varepsilon}\right)_{x}
$$

can be rewritten as

$$
\sqrt{\rho^{\varepsilon}} u_{t t}^{\varepsilon}-\left(\frac{1}{\sqrt{\rho^{\varepsilon}}} u_{x}^{\varepsilon}\right)_{x}=0,
$$

but for this type of equation we have the following general result:

Lemma 3.4. Let $\rho \in L^{\infty}(\mathbb{R})$ be a 1-periodic function satisfying (3.20). Assume that the the following coupling condition

$$
\rho(x) a(x)=K>0, \quad \text { a.e. } x \in(0,1),
$$

holds and that

$$
\left(u_{0}, \rho^{\varepsilon} u_{1}\right) \in L^{2}(0,1) \times H^{-1}(0,1) .
$$

Then, for any $T>2 \bar{\rho} / \sqrt{K}$ and $0<\varepsilon<\varepsilon_{0}$, with $\varepsilon_{0}$ small enough, there exists a control $f^{\varepsilon} \in L^{2}(0, T)$ such that the solution of problem

$$
\left(P^{\varepsilon}\right) \begin{cases}\rho^{\varepsilon}(x) u_{t t}-\left(a^{\varepsilon}(x) u_{x}^{\varepsilon}\right)_{x}=0, & 0<x<1, t>0, \\ u^{\varepsilon}(0, t)=0, \quad u^{\varepsilon}(1, t)=f(t), & t>0, \\ u^{\varepsilon}(x, 0)=u_{0}(x), \quad u_{t}^{\varepsilon}(x, 0)=u_{1}(x), & 0<x<1,\end{cases}
$$


satisfies the uniform exact controllability condition (1.2). Moreover,

$$
\begin{cases}u^{\varepsilon} \rightarrow u & \text { strongly in } L^{\infty}\left(0, T ; L^{2}(0,1)\right), \\ f^{\varepsilon} \rightarrow f \quad \text { strongly in } L^{2}(0, T),\end{cases}
$$

where $u$ and $f$ are, respectively, the state and control of the homogenized system

$$
\left(P^{0}\right) \begin{cases}\bar{\rho} u_{t t}-\frac{K}{\bar{\rho}} u_{x x}=0, & 0<x<1, t>0 \\ u(0, t)=0, \quad u(1, t)=f(t), & t>0 \\ u(x, 0)=u_{0}(x), \quad u_{t}(x, 0)=u_{1}(x), & 0<x<1 .\end{cases}
$$

Proof. Consider the function

$$
\begin{aligned}
\varphi_{\varepsilon}:[0,1] & \rightarrow \mathbb{R} \\
x & \mapsto \varphi_{\varepsilon}(x)=K^{-1 / 2} \int_{0}^{x} \rho^{\varepsilon}(s) d s .
\end{aligned}
$$

It is not hard to show that this function satisfies:

(i) $\left(\varphi_{\varepsilon}\right)^{\prime}(x)>0$ a.e. $x \in(0,1)$,

(ii) $\varphi_{\varepsilon} \in W^{1, \infty}(0,1), \varphi_{\varepsilon}^{-1} \in W^{1, \infty}\left(0, \varphi_{\varepsilon}(1)\right)$,

(iii) $\left\|\varphi_{\varepsilon}\right\|_{W^{1, \infty}(0,1)},\left\|\varphi_{\varepsilon}^{-1}\right\|_{W^{1, \infty}\left(0, \varphi_{\varepsilon}(1)\right)} \leq M$, with $M$ independent of $0<\varepsilon<1$, and

(iv) $\varphi_{\varepsilon}(x) \rightarrow \bar{\rho} K^{-1 / 2} x$ as $\varepsilon \rightarrow 0$ uniformly in $x \in[0,1]$.

With the change of variable $y=\varphi_{\varepsilon}(x)$, it is also very easy to see that the system $\left(P^{\varepsilon}\right)$ transforms into

$$
\left(P_{1}^{\varepsilon}\right) \begin{cases}v_{t t}^{\varepsilon}-v_{y y}^{\varepsilon}=0, & 0<y<\varphi_{\varepsilon}(1), t>0, \\ v^{\varepsilon}(0, t)=0, \quad v^{\varepsilon}\left(\varphi_{\varepsilon}(1), t\right)=f^{\varepsilon}(t), & t>0 \\ v^{\varepsilon}(y, 0)=u_{0}\left(\varphi_{\varepsilon}^{-1}(y)\right), \quad v_{t}^{\varepsilon}(y, 0)=u_{1}\left(\varphi_{\varepsilon}^{-1}(y)\right), & 0<y<\varphi_{\varepsilon}(1)\end{cases}
$$

which is a constant-coefficient system. We then may apply the results on the constantcoefficients wave equation, in particular the explicit formula of the control (3.17), to conclude that this system is exactly controllable at time $T^{\varepsilon}=2 \varphi_{\varepsilon}(1)$ and its control is explicitly given by

$f^{\varepsilon}(t)= \begin{cases}\frac{1}{2}\left[u_{0}\left(\varphi_{\varepsilon}^{-1}\left(\varphi_{\varepsilon}(1)-t\right)\right)+\int_{\varphi_{\varepsilon}(1)-t}^{\varphi_{\varepsilon}(1)} u_{1}\left(\varphi_{\varepsilon}^{-1}(s)\right) d s\right], & 0<t<\varphi_{\varepsilon}(1), \\ \frac{1}{2}\left[-u_{0}\left(\varphi_{\varepsilon}^{-1}\left(t-\varphi_{\varepsilon}(1)\right)\right)+\int_{t-\varphi_{\varepsilon}(1)}^{\varphi_{\varepsilon}(1)} u_{1}\left(\varphi_{\varepsilon}^{-1}(s)\right) d s\right], & \varphi_{\varepsilon}(1)<t<2 \varphi_{\varepsilon}(1),\end{cases}$

where the second term in this expression must be understood in the sense of Remark 3.2 ,

The state $u^{\varepsilon}(x, t)$ of $\left(P^{\varepsilon}\right)$ can be calculated through the solution of $\left(P_{1}^{\varepsilon}\right)$ as well. Precisely, we have that $u^{\varepsilon}(x, t)$ is the restriction to the interval $[0,1]$ of the function

$$
U^{\varepsilon}(x, t)=\frac{1}{2}\left[U_{0}^{\varepsilon}\left(\varphi_{\varepsilon}(x)-t\right)+U_{0}^{\varepsilon}\left(\varphi_{\varepsilon}(x)+t\right)+\int_{\varphi_{\varepsilon}(x)-t}^{\varphi_{\varepsilon}(x)+t} U_{1}^{\varepsilon}(s) d s\right],
$$

$U_{0}^{\varepsilon}$ and $U_{1}^{\varepsilon}$ being the extensions of the initial data of the problem $\left(P_{1}^{\varepsilon}\right)$ to $\mathbb{R}$ in an odd fashion to $\left[-\varphi_{\varepsilon}(1), 0\right]$ and with value zero elsewhere.

Similarly, the state and control of the homogenized problem $\left(P^{0}\right)$ are also explicitly given by the formulae we have for the constant-coefficients wave equation. 
Since $\varphi_{\varepsilon}(1) \rightarrow \bar{\rho} / \sqrt{K}$ as $\varepsilon \rightarrow 0$, given $T>2 \bar{\rho} / \sqrt{K}$ there exists $\varepsilon_{0}>0$ such that $2 \varphi_{\varepsilon}(1)<T$ for all $0<\varepsilon<\varepsilon_{0}$. For $0<\varepsilon<\varepsilon_{0}, 0<x<1$ and $0<t<T$, still denote by $u^{\varepsilon}(x, t)$ and $f^{\varepsilon}(t)$ the extensions of these two functions to the interval $(0, T)$ with value zero in $\left(2 \varphi_{\varepsilon}(1), T\right)$ in the time variable.

The convergence of the family of states $u^{\varepsilon}(x, t)$ and controls $f^{\varepsilon}(t)$ of the problem $\left(P^{\varepsilon}\right)$ to the state and control of $\left(P^{0}\right)$, respectively, now follows by dominated convergence, taking into account the explicit formulae we have for them and properties (iii)-(iv) of the change of variable $\varphi_{\varepsilon}$.

The proof of Theorem 3.3 is now an immediate consequence of this result. We just have to put $K=1$ and replace $\bar{\rho}$ by $\rho^{*}$.

\section{Further comments and open problems.}

- As mentioned throughout this paper, to obtain a full characterization of the class of nonresonant initial data for the control system $\left(C^{\varepsilon}\right)$ is the main problem that remains open.

- As for the possible extensions of the results of this paper to the case of dimension $N>1$ we may say the following. Since the homogenization result that Theorem 2.1 is based on still holds in the case of several space dimensions we do think that this result, and consequently Theorem 3.1, are true in higher dimensions. But, in this case we do not have an explicit formula as in (2.8) for the conormal derivatives of the solutions of the adjoint system so that we cannot directly follow the lines of the proof of Theorem 2.1. A careful analysis of these conormal derivatives is then in order. It is however worthwhile to mention here the reference [1] where a first step in this direction was given. As for Theorem 3.3, it seems that this result should also be true in the case of several space dimensions, but we now have the obstacle that in dimension $N>1$ we may not use the change of variable (3.23), which is a key factor in the proof of this result.

- The problem of lack of convergence of the controls disappears when we deal with approximate controllability instead of exact controllability. As shown by E. Zuazua in [16] for the case of the heat equation and internal control, in the case of approximate controllability the controls are uniformly bounded and they converge in $L^{2}$ to an approximate control of the associated homogenized system. The same holds true for the case of the wave equation.

Acknowledgments. We wish to thank C. Castro and E. Zuazua for some comments and suggestions on this work.

\section{REFERENCES}

[1] M. Avellaneda and F. H. Lin, Homogenization of Poisson's kernel and applications to boundary control, J. Math. Pures Appl. 68 (1989), 1-29. MR0985952 (90g:35016)

[2] M. Avellaneda, C. Bardos and J. Rauch, Contrôlabilité exacte, homogénéisation et localisation d'ondes dans un milieu non-homogène, Asymptot. Anal. 5 (1992), 481-494. MR.1169354|(93e:93004

[3] S. Brahim-Otsmane, G. A. Francfort and F. Murat, Correctors for the homogenization of the wave and heat equations, J. Math. Pures Appl. 71 (1992), 197-231. MR.1172450 (93d:35012) 
[4] C. Castro, Boundary controllability of the one-dimensional wave equation with rapidly oscillating density, Asymptot. Anal. 20 (1999), 317-350. MR.1715339 (2000i:93007)

[5] C. Castro and E. Zuazua, Concentration and lack of observability of waves in highly heterogeneous media, Arch. Rat. Mech. Anal. 164 (1) (2003), 39-72. MR1921162 (2003f:93050)

[6] C. Castro and E. Zuazua, Low frequency asymptotic analysis of a string with rapidly oscillating density, SIAM J. Appl. Math. 60 (4) (2000), 1205-1233. MR1760033 (2001h:34117)

[7] C. Castro and E. Zuazua, High frequency asymptotic analysis of a string with rapidly oscillating density, Eur. J. Appl. Math. 11 (6) (2000), 595-622. MR.1811309(2001k:34093)

[8] D. Cioranescu and P. Donato, An Introduction to Homogenization, Oxford Lectures Series in Mathematics and its Applications, 17, 1999. MR1765047 (2001j:35019)

[9] L. C. Evans, Partial Differential Equations, Graduate Studies in Mathematics, Vol. 19 AMS, 1998. MR.1625845 (99e:35001)

[10] E. Fernandez-Cara and E. Zuazua, On the null controllability of the one-dimensional heat equation with BV coefficients, Comput. Appl. Math. 21 (1) (2002) 167-190. MR2009951 (2004h:93014)

[11] G. Lebeau, The wave equation with oscillating density: Observability at low frequency, ESAIM: Control, Optimisation and Calculus of Variations, 5 (2000), 219-258. MR1750616 (2001h:35112)

[12] W. Krabs and G. Leugering, On boundary controllability of one-dimensional vibrating systems by $W_{0}^{1, p}$-controls for $p \in[2, \infty]$, Mathematical Methods in the Applied Sciences, 17 (1994), 77-93. MR 1258257 (95a:93010)

[13] J. L. Lions, Contrôlabilité exacte, perturbations et stabilisation des systèmes distribués. Tome 1, Contrôlabilité exacte, Masson, 1988. MR0953547(90a:49040)

[14] J. Necas, Les méthodes directes en théorie des équations elliptiques, Masson, 1967.

[15] D. L. Russell, Controllability and stabilizability theory for linear partial differential equations: Recent progress and open questions, SIAM Rev., 20 (4) (1978), 639-739. MR0508380 (80c:93032)

[16] E. Zuazua, Approximate controllability for linear parabolic equations with rapidly oscillating coefficients, Control and Cybernetics, 23 (4) (1994), 793-801. MR.1303384 (95k:93013)

[17] E. Zuazua, Propagation, Observation, and Control of Waves approximated by finite difference methods, SIAM Rev., 47 (2) (2005), 197-243. MR2179896 